\title{
UPPER BOUNDS FOR THE NUMBER OF NUMBER FIELDS WITH ALTERNATING GALOIS GROUP
}

\author{
ERIC LARSON AND LARRY ROLEN
}

(Communicated by Matthew A. Papanikolas)

\begin{abstract}
Let $N\left(n, A_{n}, X\right)$ be the number of number fields of degree $n$ whose Galois closure has Galois group $A_{n}$ and whose discriminant is bounded by $X$. By a conjecture of Malle, we expect that $N\left(n, A_{n}, X\right) \sim C_{n} \cdot X^{\frac{1}{2}} \cdot(\log X)^{b_{n}}$ for constants $b_{n}$ and $C_{n}$. For $6 \leq n \leq 84393$, the best known upper bound is $N\left(n, A_{n}, X\right) \ll X^{\frac{n+2}{4}}$, by Schmidt's theorem, which implies there are $\ll$ $X^{\frac{n+2}{4}}$ number fields of degree $n$. (For $n>84393$, there are better bounds due to Ellenberg and Venkatesh.) We show, using the important work of Pila on counting integral points on curves, that $N\left(n, A_{n}, X\right) \ll X^{\frac{n^{2}-2}{4(n-1)}+\epsilon}$, thereby improving the best previous exponent by approximately $\frac{1}{4}$ for $6 \leq n \leq 84393$.
\end{abstract}

\section{Introduction AND STATEMENT OF RESUlts}

For any positive integers $n$ and $X$ and for any fixed transitive permutation group $G$, we would like to count $N(n, G, X)$, defined to be the number of degree $n$ number fields $K$ whose Galois closure has Galois group $G$ and for which $\left|D_{K}\right| \leq X$. Furthermore, let $N(n, X)$ denote the number of all degree $n$ number fields with discriminant bounded in absolute value by $X$. It is an old conjecture, sometimes attributed to Linnik, that

$$
N(n, X) \sim c_{n} X \quad(n \text { fixed, } X \rightarrow \infty) .
$$

The conjecture is trivial when $n=2$, and was proven for $n=3$ by Davenport and Heilbronn 4 and for $n=4,5$ by Bhargava 1 , 2]. For all but finitely many $n$, the current best upper bound, due to Ellenberg and Venkatesh in [5], states:

$$
N(n, X) \ll\left(X \cdot B_{n}\right)^{\exp (C \log \sqrt{n})} .
$$

Here, $B_{n}$ depends only on $n$ and $C$ is an absolute constant. For large $n$, the bound of Ellenberg and Venkatesh is the lowest. For $6 \leq n \leq 84393$, however, the best bound is due to Schmidt [8] :

$$
N(n, X) \ll X^{\frac{n+2}{4}} .
$$

In this note, we study the case when $G=A_{n}$. By a conjecture of Malle ([]. $)$, we expect that

$$
N\left(n, A_{n}, X\right) \stackrel{?}{\sim} c(n) \cdot X^{\frac{1}{2}} \cdot(\log X)^{b(n)-1},
$$

Received by the editors July 6, 2011.

2010 Mathematics Subject Classification. Primary 11R99, 11G99.

The authors are grateful for the support of the NSF in funding the Emory 2011 REU. The authors would like to thank our advisor, Andrew Yang, as well as Ken Ono for their guidance, useful conversations, improving the quality of exposition of this article, and hosting the REU. 
for some constant $c(n)$ and an explicit constant $b(n)$. We improve Schmidt's general bound when applied to $A_{n}$. In particular, we can use Pila's results on counting integral points on geometrically irreducible curves [7] to show the following:

Theorem 1.1. We have

$$
N\left(n, A_{n}, X\right) \ll X^{\frac{n^{2}-2}{4(n-1)}} \cdot \log (X)^{2 n+1},
$$

where the implied constant depends only on $n$.

Remark 1.2. Throughout this note, we write $f \ll g$ to mean that $f \leq c \cdot g$ for a constant $c$ depending only on the degree of the number field (or degree of the algebraic variety) in question.

Note that the exponent improves on the previous record by a power of $X$ of about $\frac{1}{4}$ for $6 \leq n \leq 84393$. The method uses point counting on varieties in a similar manner as in 5]. The improvement follows from viewing these varieties as fibrations of curves, controlling the fibers which are not geometrically irreducible, and using a bound of Pila on counting integral points on geometrically irreducible curves. The case when $n$ is even is easier, and in the odd case we proceed by using basic theorems on covering spaces.

\section{UPPER BOUNDS VIA POINT COUNTING}

If $K$ is a number field of discriminant $D_{K}$ and degree $n$, then Minkowski theory implies that there is an element $\alpha \in \mathcal{O}_{K}$ of trace zero with

$$
|\alpha| \ll D_{K}^{\frac{1}{2(n-1)}} \quad \text { (under any Archimedean valuation), }
$$

where the implied constant depends only on $n$.

When $\operatorname{Gal}\left(K^{\text {gal }} / \mathbb{Q}\right) \simeq A_{n}, K$ must be a primitive extension of $\mathbb{Q}$, so $K=\mathbb{Q}(\alpha)$ and the characteristic polynomial of $\alpha$ will determine $K$. One can use this to give an upper bound on $N\left(n, A_{n}, X\right)$. To see this, note that every pair $(K, \alpha)$ as above gives a $\mathbb{Z}$-point of $\operatorname{Spec} R$, for

$$
R=\mathbb{Z}\left[x_{1}, x_{2}, \ldots, x_{n}\right]^{A_{n}} /\left(s_{1}\right), \quad \text { where } \quad s_{1}=x_{1}+x_{2}+\cdots+x_{n} .
$$

(Here $\mathbb{Z}\left[x_{1}, x_{2}, \ldots, x_{n}\right]^{A_{n}}$ denotes the ring of $A_{n}$-invariants in $\mathbb{Z}\left[x_{1}, x_{2}, \ldots, x_{n}\right]$.) It is a classical theorem that the ring of $A_{n}$-invariant functions is generated by the symmetric functions and the square root of the discriminant; i.e., we have

$$
\mathbb{Z}\left[x_{1}, x_{2}, \ldots, x_{n}\right]^{A_{n}} \simeq \mathbb{Z}\left[s_{1}, s_{2}, \ldots, s_{n}, D\right] /\left(D^{2}=\operatorname{Disc}\left(t^{n}-s_{1} t^{n-1}+\cdots \pm s_{n}\right)\right),
$$

so therefore

$$
R \simeq \mathbb{Z}\left[s_{2}, \ldots, s_{n}, D\right] /\left(D^{2}=\operatorname{Disc}\left(t^{n}+s_{2} t^{n-2}+\cdots \pm s_{n}\right)\right) .
$$

Thus, to give an upper bound on $N\left(n, A_{n}, X\right)$, it suffices to bound the number of $\mathbb{Z}$-points of Spec $R$ which satisfy the inequalities

$$
\left|s_{j}\right| \ll X^{\frac{j}{2(n-1)}} \quad \text { and } \quad|D| \ll X^{\frac{n}{4}} .
$$




\section{Proof of Theorem 1.1 when $n$ IS EVEN}

When $n$ is even, Theorem 1.1 is relatively straightforward; therefore, we begin by examining this case.

Proposition 3.1. Theorem 1.1 holds when $n$ is even.

Proof. By fixing $s_{2}, s_{3}, \ldots, s_{n-1}$, we can view $\operatorname{Spec} R$ as a fibration of plane curves over $\mathbb{A}^{n-2}$. Each of these curves is then the zero locus of a polynomial of the form

$$
D^{2}=\text { a polynomial of odd degree in } s_{n} \text {. }
$$

In particular, these curves are geometrically irreducible. Therefore, we can apply Pila's bound [7, which states that the number of integral points on a geometrically irreducible plane curve of degree $d$ whose coordinates are bounded in absolute value by $B$ is at most

$$
(3 d)^{4 d+8} \cdot B^{\frac{1}{d}} \cdot(\log B)^{2 d+3} \ll B^{\frac{1}{d}} \cdot(\log B)^{2 d+3} .
$$

For the curves defined by (2), we seek to count integral points with

$$
\left|s_{n}\right| \ll X^{\frac{n}{2(n-1)}} \quad \text { and }|D| \ll X^{\frac{n}{4}} .
$$

By Pila's result above, the number of such points is

$$
\ll\left(X^{\frac{n}{4}}\right)^{\frac{1}{n-1}} \cdot\left(\log \left(X^{\frac{n}{4}}\right)\right)^{2 \cdot(n-1)+3} \ll X^{\frac{n}{4(n-1)}} \cdot(\log X)^{(2 n+1)} .
$$

Therefore, using the bounds (1) on the other $s_{j}$ from the previous section, we have

$$
N\left(n, A_{n}, X\right) \ll\left(\prod_{j=2}^{n-1} X^{\frac{j}{2(n-1)}}\right) \cdot X^{\frac{n}{4(n-1)}} \cdot(\log X)^{2 n+1}=X^{\frac{n^{2}-2}{4(n-1)}} \cdot(\log X)^{2 n+1} .
$$

\section{Proof of Theorem 1.1 when $n$ IS ODD}

In the case when $n$ is odd, the argument of Proposition 3.1 breaks down because the curves in the fibration do not have to be geometrically irreducible. In order to circumvent this difficulty, we will show in this section that "most" of the fibers of the map $\operatorname{Spec} R \rightarrow \mathbb{A}^{n-2}$ are geometrically irreducible. We will then bound the number of integral points on the fibers that fail to be geometrically irreducible.

Definition 4.1. We say that two polynomials $f, g \in \mathbb{C}[z]$ are equivalent if $f(z)=$ $g(a z+b)$ for some $a \in \mathbb{C}^{\times}$and $b \in \mathbb{C}$.

Definition 4.2. We say that $c$ is a critical value of a polynomial $f$ if $c=f(d)$ for some $d$ with $f^{\prime}(d)=0$.

Lemma 4.3. Fix a finite set of points $S \subset \mathbb{C}$ and an integer $d$. Then there are finitely many equivalence classes of polynomials of degree $d$ whose set of critical values is contained in $S$.

Proof. Write $S=\left\{z_{1}, z_{2}, \ldots, z_{n}\right\}$, and fix some $z_{0} \notin S$. Then any polynomial of degree $d$ whose set of critical values is contained in $S$ gives rise to a map

$$
f^{*}: \pi_{1}(\mathbb{C}-S) \rightarrow \operatorname{Aut}\left(f^{-1}\left(z_{0}\right)\right) \simeq S_{d} .
$$

Since $S$ is finite, $\pi_{1}(\mathbb{C}-S)$ is finitely generated; moreover, $S_{d}$ is finite, so there are only finitely many possibilities for $f^{*}$. 
Thus, it suffices to show that any two polynomials $f$ and $g$ for which $f^{*}=g^{*}$ are equivalent. But the classical theory of covering spaces implies that when $f^{*}=g^{*}$, then $f$ and $g$ must differ by a deck transformation, which must be analytic because $f$ and $g$ are analytic coverings. The desired conclusion then follows from the wellknown fact that any automorphism of $\widehat{\mathbb{C}}$ fixing $\infty$ is of the form $z \mapsto a \cdot z+b$ with $a \in \mathbb{C}^{\times}$and $b \in \mathbb{C}$.

Lemma 4.4. Let $n$ be an integer. For any monic polynomial $p(z) \in \mathbb{C}[z]$ of degree $n-1$, there are only finitely many values of $\left(a_{2}, a_{3}, \ldots, a_{n-1}\right) \in \mathbb{C}^{n-2}$ such that $p(z)$ is the discriminant of the polynomial

$$
q(t)=t^{n}+a_{2} t^{n-2}+\cdots+a_{n-1} t-z .
$$

Proof. In order for $p(z)$ to be the discriminant of $q(t)$, every root $r$ of $p(z)$ must be (with multiplicity) a critical value of the polynomial $q_{0}(t)=t^{n}+a_{1} t^{n-1}+\cdots+a_{n-1} t$. Since $q_{0}$ is a polynomial of degree $n$, it has $n-1$ critical values (counted with multiplicity); since $p(z)$ is a polynomial of degree $n-1$, it has $n-1$ zeros (counted with multiplicity). Therefore, every critical value of $q_{0}$ is a root of $p(z)$. This completes the proof by Lemma 4.3 .

Lemma 4.5. The locus of $\left(s_{2}, s_{3}, \ldots, s_{n-1}\right) \in \mathbb{A}^{n-2}$ such that the plane curve

$$
x^{2}=\operatorname{Disc}\left(t^{n}+s_{2} t^{n-2}+\cdots \pm s_{n-1} t-y\right)
$$

fails to be geometrically irreducible is an affine variety of dimension at most $\frac{n-1}{2}$.

Proof. The corresponding plane curve fails to be geometrically irreducible if and only if the polynomial

$$
p(y)=\operatorname{Disc}\left(t^{n}+s_{2} t^{n-2}+\cdots \pm s_{n-1} t-y\right)
$$

is a perfect square. But the coefficients of $p(y)$ are regular functions in $s_{2}, s_{3}, \ldots, s_{n-1}$. Moreover, the map $\mathbb{A}^{n-2} \rightarrow \mathbb{A}^{n-1}$ induced by these regular functions is a finite map by Lemma 4.4

Since the locus of $\left(b_{1}, b_{2}, \ldots, b_{n-1}\right) \in \mathbb{A}^{n-1}$ such that $t^{n-1}+b_{1} t^{n-2}+\cdots+b_{n-1}$ is a perfect square is a Zariski-closed set of dimension $\frac{n-1}{2}$, this completes the proof.

Using the above lemma together with the ideas from Section 3 we can complete the proof of Theorem 1.1

Proof of Theorem 1.1 for $n$ odd. When $n=3$, this follows from a result of Wright [9], and when $n=5$, this follows from a result of Bhargava [2]. Thus, we can assume $n \geq 7$.

Again, we consider the fibration $\operatorname{Spec} R \rightarrow \mathbb{A}^{n-2}$ given by fixing $s_{2}, s_{3}, \ldots, s_{n-1}$. The argument given in Lemma 3.1 implies that the number of integral points lying on the geometrically irreducible fibers satisfies the required bound; it remains to see that the number of integral points lying on the geometrically reducible fibers also satisfies the required bound.

To prove this, we first note that by Lemma 4.5, all such points are contained in a subvariety of Spec $R$ of dimension at most $\frac{n-1}{2}+1=\frac{n+1}{2}$. Moreover, the projection map Spec $R \rightarrow \mathbb{A}^{n-1}$ given by fixing $s_{2}, s_{3}, \ldots, s_{n}$ is finite, so it suffices to bound the number of integral points in the box

$$
\left|s_{j}\right| \leq X^{\frac{j}{2(n-1)}}
$$


lying in a particular affine variety of dimension $\frac{n+1}{2}$. But the number of such points can be bounded by the product of the $\frac{n+1}{2}$ largest sides of the box, and therefore is

$$
\ll \prod_{j=\frac{n+1}{2}}^{n} X^{\frac{j}{2(n-1)}}=X^{\frac{(3 n+1)(n+1)}{16(n-1)}},
$$

and therefore satisfies the required bound as long as $n \geq 7$.

\section{References}

[1] M. Bhargava. The Density of Discriminants of Quartic Rings and Fields. Ann. of Math. (2) 162 (2005), no. 2, 1031-1063. MR2183288(2006m:11163)

[2] The Density of Discriminants of Quintic Rings and Fields. Ann. of Math. (2) 172 (2010), no. 3, 1559-1591. MR2745272 (2011k:11152)

[3] H. Cohen. A Survey of Discriminant Counting. Algorithmic Number Theory (Sydney, 2002), Springer, 2002, 80-94. MR2041075 (2005a:11173)

[4] H. Davenport and H. Heilbronn. On the Density of Discriminants of Cubic Fields II. Proc. Roy. Soc. London Ser. A 322 (1971), no. 1551, 405-420. MR0491593 (58:10816)

[5] J. Ellenberg and A. Venkatesh. The Number of Extensions of a Number Field with Fixed Degree and Bounded Discriminant. Ann. of Math. (2) 163 (2006), no. 2, 723-741. MR2199231 (2006j:11159)

[6] G. Malle. On the Distribution of Galois Groups II. J. Number Theory 92 (2002), no. 2, 315329. MR1884706 (2002k:12010)

[7] J. Pila. Density of Integer Points on Plane Algebraic Curves. Internat. Math. Res. Notices 1996, no. 18, 903-912. MR1420555 (97m:11126)

[8] W. M. Schmidt. Number Fields of Given Degree and Bounded Discriminant. Columbia University Number Theory Seminar (New York, 1992), Astérisque 228 (1995), 189-195. MR1330934 (96e:11153)

[9] D. Wright. Distribution of Discriminants of Abelian Extensions. Proc. London Math. Soc. (3) 58 (1989), no. 1, 17-50. MR969545 (90b:11115)

Department of Mathematics, Harvard University, Cambridge, Massachusetts 02138

E-mail address: elarson3@gmail.com

Department of Mathematics and Computer Science, Emory University, Atlanta, GEORGIA 30322

E-mail address: larry.rolen@mathcs.emory.edu 